\title{
The diagnostic value of endothelial function as a potential sensor of fatigue in health
}

This article was published in the following Dove Press journal:

Vascular Health and Risk Management

6 March 2010

Number of times this article has been viewed

\section{Yoshiko Ohnol,2 \\ Teruto Hashiguchi' \\ Ryuichi Maenosono' \\ Hidetoshi Yamashita ${ }^{3}$ \\ Yukio Taira ${ }^{3}$ \\ Kazufumi Minowa ${ }^{3}$ \\ Yoshihito Yamashita ${ }^{3}$ \\ Yuko Kato ${ }^{3}$ \\ Ko-ichi Kawahara' \\ Ikuro Maruyama' \\ 'Department of Laboratory and Vascular Medicine, Kagoshima University Graduate School of Medical and Dental Sciences, Kagoshima City, Kagoshima Prefecture, Japan; ${ }^{2}$ Department of Community Health Nursing/Nursing Informatics, School of Health Sciences, Faculty of Medicine, Kagoshima University, Kagoshima City, Kagoshima Prefecture, Japan; ${ }^{3}$ Kagoshima Seikyo General Hospital, Kagoshima City, Kagoshima Prefecture, Japan}

Correspondence: Teruto Hashiguchi Kagoshima University Graduate School of Medical and Dental Sciences, 8-35-I Sakuragaoka, Kagoshima City, Kagoshima Prefecture, 890-8520, Japan

$\mathrm{Tel}+8 \mathrm{I} 992755437$

Fax +81992752629

Email terutoha@m3.kufm.kagoshima-u.ac.jp
Purpose: Many epidemiological research studies have shown that vital exhaustion and psychosocial factors are associated with the occurrence of cerebrocardiovascular disease (CCVD). Fatigue is thought to induce endothelial dysfunction and may be linked to the occurrence of CCVD; however, no studies have investigated this potential link. We studied to determine the effect of fatigue on endothelial function in healthy subjects with no traditional CCVD risk factors or potential confounding factors to be controlled.

Subjects and methods: Peripheral arterial tonometry (PAT) was used to evaluate endothelial function. The influence of the following parameters on endothelial function was analyzed in 74 office workers without traditional CCVD risk factors at health check-ups: endothelial function before and after work, subjective fatigue, lifestyle factors such as sleeping time, and psychosocial factors such as depression and social support.

Results: Twenty-five subjects (33.8\%) had low endothelial function; reactive hyperemia (RH)-PAT index $<1.67$, even though no abnormalities were reported in the health check-ups. There was no significant difference in endothelial function before versus after labor. Of note, endothelial function was associated with the individual's level of subjective fatigue $(t=2.98, P=0.008)$ and showed a daily fluctuation, sometimes to a pathological degree $(<1.67)$.

Conclusion: We showed that, even in healthy people, endothelial function fluctuates diurnally, with an interaction between the individual's cognitive fatigue and the environment, sometimes to a pathological degree. Based on these findings, we suggest that endothelial function is an objective assessment tool of fatigue in healthy individuals.

Keywords: cerebrocardiovascular disease, endothelial dysfunction, subjective fatigue, flow-mediated dilatation, health education, reactive hyperemia peripheral arterial tonometry

\section{Introduction}

A link between increased risk of burnout or vital exhaustion and cardiovascular disease has been revealed. ${ }^{1,2}$ Furthermore, long-term fatigue or vital exhaustion was reported to be associated with cerebrocardiovascular disease (CCVD) such as cerebral infarction and sudden cardiac events in several epidemiological studies. ${ }^{3,4}$ In addition to vital exhaustion, psychosocial factors such as depression, anxiety and social support are associated with CCVD. ${ }^{5-7}$ Fatigue is thought to induce endothelial dysfunction and to be linked to the occurrence of CCVD. However, to our knowledge, no studies have investigated this potential link.

Endothelial dysfunction has been reported to increase the risk of CCVD. ${ }^{8-10}$ Endothelial cells maintain a relaxed vascular tone, achieved in part by releasing mediators such as nitric oxide. ${ }^{11}$ Endothelial cells also modulate biological processes related to the walls of the blood vessels, such as the regulation of prothrombotic and antithrombotic factors,

submit your manuscript | www.dovepress.coin 
and play a critical role in vascular homeostasis. ${ }^{12,13}$ Therefore, endothelial function is considered to be one of a marker for vascular health condition ${ }^{8,18}$ Factors known to affect endothelial function are diverse and range from traditional cardiac risk factors such as age, sex, hypertension, insulin-resistance and high cholesterol and lifestyle factors, to novel risk factors such as air pollution. ${ }^{14-16}$ Nevertheless, in addition to the many risk factors described above, the presence of hitherto unknown factors cannot be ignored. ${ }^{8}$

With increased understanding of the numerous roles of the endothelium, several novel techniques have been proposed to evaluate endothelial cell function. The assessment of endothelial function in coronary arteries is clinically significant as a predictor of future cardiovascular events. ${ }^{17}$ In recent years, noninvasive methods have been developed for screening large populations. ${ }^{14}$ The measurement of flow-mediated dilatation (FMD) is noninvasive and reflects endothelium-dependent vasodilation. ${ }^{18}$ Peripheral arterial tonometry (PAT), which was applied for the same concept as FMD, does not require skilled operators to perform this test. ${ }^{19-21}$ PAT analysis is conducted using a computerized automated algorithm and there is no inter-observer variability.
Fatigue is generally considered to be one of the most important feelings in daily life because it is closely related to quality of life and well-being. However, fatigue is a complex phenomenon that involves a number of physical, psychosocial, and behavioral processes associated with multidimensional concepts. ${ }^{22,23}$ The perception of fatigue can differ between people and, even within one person, the meaning of fatigue can change. ${ }^{24}$ Many tools have been developed to measure fatigue in nonclinical and medical settings, but it is difficult to define fatigue and there is no gold-standard method to assess fatigue. ${ }^{25}$ Thus, in terms of CCVD onset, the development of an objective measure of fatigue is an important focus of research.

In this report, we analyzed endothelial function in healthy subjects without classical risk factors for CCVD, and evaluated the potential for endothelial function to be used as an objective measurement of fatigue in healthy people.

\section{Material and methods \\ Study design and subjects}

Four hundred and eleven subjects were recruited from the entire employee population of Kagoshima Seikyo General Hospital (Figure 1). All participants were aged at

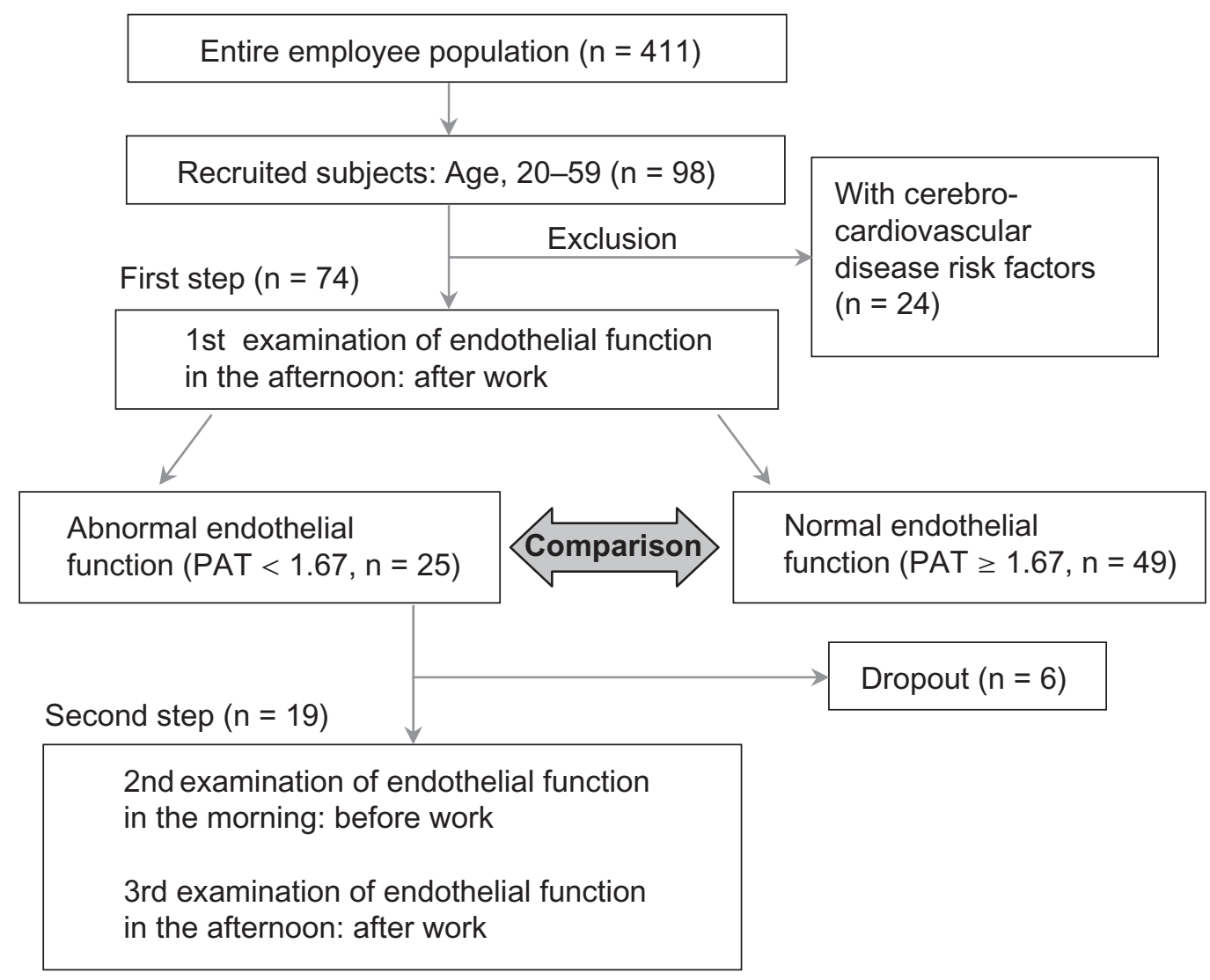

Figure I Study protocol.

Abbreviations: PAT, peripheral arterial tonometry; $\mathrm{n}$, number. 
least 20 years. Health check-up data were collected from 411 subjects to select subjects who were judged to have normal health status. We excluded individuals with potentially confounding health conditions, including pregnancy, breast feeding, currently taking lipid-lowering medication, history of severe conditions (eg, chronic fatigue syndrome, coronary artery disease, cerebrovascular disease, heart failure, diabetes, renal impairment, uncontrolled hypertension), untreated or clinically evident thyroid disease, anemia, dietary deficiencies, valvular heart disease, adrenal conditions, and individuals on medications. From these subjects, further analysis was conducted on 74 subjects from whom informed consent was obtained for the additional procedures. These 74 subjects were instructed not to change their lifestyle, including routine diet, exercise or weight management, and working or rest habits while enrolled in the study. The characteristics of the 74 subjects are shown in Table 1. Twenty-seven males (aged 23-59 years) and 47 females (aged 24-59 years) completed all evaluations. Overall, data for six factors (Table 2), including blood examination, were collected for each subject. The study was approved by Kagoshima Seikyo General Hospital Institutional Review Board (serial No.10) in 2007 and was conducted according to the principles of the Declaration of Helsinki. All participants provided written informed consent.

\section{First step}

Endothelial function was assessed by PAT; this value is referred to as the first measurement of endothelial function
(Figure 1). All of the examinations were conducted while the subjects rested in the supine position, in a quiet, dim, and temperature- and humidity-controlled room $\left(22 \pm 2{ }^{\circ} \mathrm{C}\right.$, 40\%-60\%). Brachial-ankle pulse wave velocity (ba-PWV) and ankle-brachial index (ABI) were assessed using a volume-plethysmograph (Form/ABI; Colin, Co Ltd, Aichi, Japan). Fatigue, psychosocial factors and lifestyle were assessed by questionnaire. Lifestyle factors were assessed using Breslow's seven health habits questionnaire, which covered smoking, drinking, exercise, sleeping of 7-8 hours per day, daily breakfast and eating between meals. ${ }^{26}$

\section{Second step}

A PAT index value $<1.67$, which indicates low endothelial function, was observed in 25 subjects. Subjects with abnormal results in the first examination after their daily work underwent a second examination. Re-evaluation of the endothelial function was conducted in 19 of these 25 subjects (six subjects dropped out). In these 19 subjects, PAT (second and third assessments of endothelial function), ba-PWV and ABI were assessed twice a day; before work in the morning and after work in the afternoon (Figure 1).

\section{The PAT system}

Endothelial function was measured noninvasively by PAT (Endo-PAT2000; Itamar Medical Ltd, Caesarea, Israel) using a finger plethysmographic device, which enables isolated detection of changes in pulsatile arterial volume.

Table I Characteristics of the subjects

\begin{tabular}{llll}
\hline & Mean \pm SD & Male & Female \\
\hline $\mathrm{n}$ & 74 & 27 & 47 \\
Age (years) & $38.3 \pm 9.54$ & $39.7 \pm 8.93$ & $37.5 \pm 9.86$ \\
RH-PAT index & $1.95 \pm 0.47$ & $1.84 \pm 0.43$ & $2.02 \pm 0.49$ \\
Systolic blood pressure* $(\mathrm{mmHg})$ & $113.7 \pm 14.16$ & $121.3 \pm 12.40$ & $109.3 \pm 13.82$ \\
Diastolic blood pressure* $(\mathrm{mmHg})$ & $68.0 \pm 11.43$ & $74.3 \pm 10.17$ & $64.3 \pm 10.56$ \\
Pulse pressure (mmHg) & $45.7 \pm 7.98$ & $47.4 \pm 8.49$ & $45.1 \pm 7.40$ \\
Heart rate (beat/min) & $62.2 \pm 9.43$ & $62.4 \pm 12.12$ & $62.4 \pm 7.85$ \\
Body mass index* $\left(\mathrm{kg} / \mathrm{m}^{2}\right)$ & $21.7 \pm 3.40$ & $23.0 \pm 3.68$ & $21.0 \pm 3.02$ \\
Smoking* (Yes/No) & $20 / 54$ & $13 / 14$ & $7 / 40$ \\
Habitual drinking*(Yes/No) & $9 / 65$ & $9 / 18$ & $0 / 47$ \\
Habitual exercise (Yes/No) & $18 / 56$ & $6 / 21$ & $12 / 35$ \\
Habitual sleeping; $7-8$ hrs (Yes/No) & $44 / 30$ & $18 / 9$ & $26 / 2 \mid$ \\
Habitual daily breakfast (Yes/No) & $63 / 11$ & $24 / 3$ & $39 / 8$ \\
Habitual eating between meals*(Yes/No) & $45 / 29$ & $12 / 15$ & $33 / 14$ \\
\hline
\end{tabular}

Notes: Data are presented as means \pm standard deviation or number. $* P<0.05$ vs Male group.

Abbreviations: RH-PAT, reactive hyperemia peripheral arterial tonometry; $\mathrm{n}$, number. 
Table 2 Comparison of characteristics between normal and low response of endothelial function

\begin{tabular}{|c|c|c|c|}
\hline & $\begin{array}{l}\text { Normal response } \\
\text { group }(\mathrm{RH} \geq \mathrm{I.67})\end{array}$ & $\begin{array}{l}\text { Low response } \\
\text { group }(\mathbf{R H}<\mathbf{1 . 6 7 )}\end{array}$ & $P$ value \\
\hline $\mathrm{n}$ & 49 & 25 & \\
\hline Female subjects & $36(72.0 \%)$ & $14(53.8 \%)$ & $0.073 \mathrm{NS}$ \\
\hline Postmenopausal subjects & $2(4.1 \%)$ & $2(8.0 \%)$ & $0.48 \mathrm{I} N \mathrm{NS}$ \\
\hline Age (years) & $38.7 \pm 9.8$ & $39.8 \pm 9.9$ & $0.648 \mathrm{NS}$ \\
\hline Body mass index $\left(\mathrm{kg} / \mathrm{m}^{2}\right)$ & $21.4 \pm 3.3$ & $22.4 \pm 3.6$ & $0.100 \mathrm{NS}$ \\
\hline Family history of CCVD & $4(8.2 \%)$ & $3(12.0 \%)$ & $0.460 \mathrm{NS}$ \\
\hline \multicolumn{4}{|l|}{ Lifestyle } \\
\hline Smoking & II (22.4\%) & $9(36.0 \%)$ & $0.218 \mathrm{NS}$ \\
\hline Habitual drinking & $5(10.2 \%)$ & $4(16.4 \%)$ & $0.476 \mathrm{NS}$ \\
\hline Habitual exercise & $13(26.5 \%)$ & $5(20.0 \%)$ & $0.538 \mathrm{NS}$ \\
\hline Habitual sleeping; 7-8 hours & $30(61.2 \%)$ & $14(56.0 \%)$ & $0.667 \mathrm{NS}$ \\
\hline Habitual daily breakfast & $44(89.8 \%)$ & $19(76.0 \%)$ & $0.117 \mathrm{NS}$ \\
\hline Habitual eating between meals & $32(65.3 \%)$ & $13(52.0 \%)$ & $0.271 \mathrm{NS}$ \\
\hline \multicolumn{4}{|l|}{ Psychosocial factors } \\
\hline Self-rating depression scale & $44.3 \pm 7.9$ & $44.0 \pm 10.6$ & $0.929 \mathrm{NS}$ \\
\hline State-trait anxiety inventory & $50.4 \pm 10.4$ & $50.2 \pm 11.2$ & $0.95 \mathrm{I} N \mathrm{NS}$ \\
\hline Self-esteem scale & $5.1 \pm 2.4$ & $5.6 \pm 2.9$ & $0.495 \mathrm{NS}$ \\
\hline Self-repression scale & $\mid \mathrm{I} .1 \pm 3.6$ & $9.8 \pm 3.2$ & $0.201 \mathrm{NS}$ \\
\hline \multicolumn{4}{|l|}{ Emotional social-support } \\
\hline support from family & $7.1 \pm 3.6$ & $7.5 \pm 3.2$ & $0.670 \mathrm{NS}$ \\
\hline support from friends & $7.1 \pm 3.3$ & $7.2 \pm 3.5$ & $0.855 \mathrm{NS}$ \\
\hline \multicolumn{4}{|l|}{ Blood Examination } \\
\hline Leukocyte $\left(\mathrm{n} / \mathrm{mm}^{3}\right)$ & $6317.7 \pm 1180.2$ & $6660.7 \pm 1773.0$ & 0.482 NS \\
\hline Erythrocyte $\left(\mathrm{n} \times 10^{4} / \mathrm{mm}^{3}\right)$ & $478.5 \pm 31.2$ & $463.4 \pm 40.6$ & $0.133 \mathrm{NS}$ \\
\hline Hematocrit (\%) & $45.0 \pm 3.2$ & $41.9 \pm 8.3$ & $0.146 \mathrm{NS}$ \\
\hline Platelet $\left(\mathrm{n} \times 10^{4} / \mathrm{mm}^{3}\right)$ & $23.4 \pm 5.7$ & $24.5 \pm 4.9$ & $0.516 \mathrm{NS}$ \\
\hline Post-prandial plasma glucose (mg/dl) & $101.4 \pm 22.4$ & $103.1 \pm 14.1$ & $0.815 \mathrm{NS}$ \\
\hline Hemoglobin A Ic (\%) & $5.1 \pm 0.3$ & $5.0 \pm 0.5$ & $0.198 \mathrm{NS}$ \\
\hline \multicolumn{4}{|l|}{ Serum level of biliary enzymes (IU/I) } \\
\hline Alkali Phosphatase & $216.6 \pm 53.6$ & $216.1 \pm 58.6$ & $0.976 \mathrm{NS}$ \\
\hline$\gamma$ Guanosine triphosphate & $32.9 \pm 29.6$ & $30.3 \pm 28.9$ & $0.738 \mathrm{NS}$ \\
\hline Glutomic oxaloacetic transaminase & $21.3 \pm 6.3$ & $20.4 \pm 6.6$ & $0.623 \mathrm{NS}$ \\
\hline Glutamate pyruvate transaminase & $21.8 \pm 14.0$ & $21.0 \pm 14.0$ & $0.837 \mathrm{NS}$ \\
\hline Serum creatinine $(\mathrm{mg} / \mathrm{dl})$ & $0.77 \pm 0.2$ & $0.76 \pm 0.2$ & $0.862 \mathrm{NS}$ \\
\hline Uric acid (mg/dl) & $5.4 \pm 1.4$ & $4.9 \pm 1.2$ & $0.211 \mathrm{NS}$ \\
\hline HDL cholesterol (mg/dl) & $62.1 \pm 13.2$ & $67.8 \pm 17.3$ & $0.181 \mathrm{NS}$ \\
\hline LDL cholesterol (mg/dl) & $113.4 \pm 25.4$ & $113.7 \pm 27.4$ & $0.969 \mathrm{NS}$ \\
\hline Triglyceride $(\mathrm{mg} / \mathrm{dl})$ & $104.2 \pm 56.6$ & $97.7 \pm 61.2$ & $0.680 \mathrm{NS}$ \\
\hline \multicolumn{4}{|l|}{ Blood dynamics } \\
\hline Systolic blood pressure $(\mathrm{mmHg})$ & $115.2 \pm 10.7$ & $113.9 \pm 15.9$ & $0.711 \mathrm{NS}$ \\
\hline Diastolic blood pressure $(\mathrm{mmHg})$ & $69.2 \pm 10.6$ & $68.0 \pm 12.1$ & $0.667 \mathrm{NS}$ \\
\hline Right ba-PWV (cm/sec) & $1228.5 \pm 184.0$ & $1183.8 \pm 182.7$ & $0.317 \mathrm{NS}$ \\
\hline Leftt ba-PWV $(\mathrm{cm} / \mathrm{sec})$ & $1232.1 \pm 159.9$ & $1201.2 \pm 176.5$ & $0.458 \mathrm{NS}$ \\
\hline Right Ankle-brachial Index & $1.07 \pm 0.1$ & $1.10 \pm 0.1$ & $0.385 \mathrm{NS}$ \\
\hline
\end{tabular}


Table 2 (Continued)

\begin{tabular}{llll}
\hline & Normal response & Low response & P value \\
& group $(\mathbf{R H} \geq \mathbf{I . 6 7})$ & group $(\mathbf{R H}<\mathbf{1 . 6 7 )}$ & $0.317 \mathrm{NS}$ \\
\hline Left ankle-brachial Index & $1.09 \pm 0.1$ & $1.11 \pm 0.9$ & $0.966 \mathrm{NS}$ \\
Pulse pressure $(\mathrm{mmHg})$ & $46.0 \pm 6.3$ & $45.9 \pm 8.6$ & $0.273 \mathrm{NS}$ \\
Heart rate $(\mathrm{n} / \mathrm{min})$ & $64.1 \pm 11.7$ & $61.6 \pm 8.1$ & \\
\hline
\end{tabular}

Notes: Data are presented as means \pm standard deviation or number. $* P<0.05$ vs Low response group.

Abbreviations: RH-PAT, reactive hyperemia peripheral arterial tonometry; n, number; HDL, high-dsensity lipoproteinc; LDL, low-density lipoprotein; ba-PWV, brachial ankle pulse wave velocity; CCVD, cerebro-cardiovascular disease including hypertension.

The device consists of two finger-mounted cuffs, which comprise inflatable Neoprene air-cushions within a rigid external case. The PAT data were analyzed by a computer in an operator-independent manner. The reproducibility of the PAT measurements were verified using previously reported statistical procedures. ${ }^{21}$ PAT is also configured to unload arterial wall tension and increase the range of arterial wall motion without inducing potentially confounding vasomotor changes. A value of $<1.67$ is considered to indicate endothelial dysfunction, as determined in a population at risk for ischemic heart disease. ${ }^{20,28}$ Therefore, in this study, we allocated subjects with an PAT index value of $<1.67$ to a low-response group, and those with index values of $\geq 1.67$ to the normal-response group. In accordance with a study by Bland and Altman, the parameters of endothelial function showed repeatability within two standard deviations (SD) and are reproducible. ${ }^{29}$

\section{Assessment of fatigue and psychosocial factors}

We used the following four methods to assess fatigue and psychosocial factors. a) To evaluate the individual's level of the relative subjective fatigue, we asked the subject to compare their subjective fatigue twice a day at the second and third measurements of endothelial function. The individual's level of subjective fatigue was subjectively classified into two groups according to the subjects' own judgments: less fatigue and more fatigue. The relative level of subjective fatigue was evaluated without informing the subjects of their PAT values. b) Evaluation of the strength of conventionally quantified fatigue: The Ministry of Health in Japan, Labor and Welfare's accumulated fatigue checklist (AFC) ${ }^{30}$ was administered at the second measurement of endothelial function to evaluate the strength of quantified fatigue. The AFC was evaluated without informing the subjects of their PAT values. c) Evaluation of sleeping duration: The subjects were asked to report their duration of sleeping on the day prior to the study day. d) Evaluation of psychosocial factors: Psychosocial factors were evaluated by the 20-item Self-Rated Depression Scale, ${ }^{31}$ the 10 -item Self-Esteem Scale, ${ }^{32}$ the 20 -item State-Trait Anxiety Inventory, ${ }^{33}$ the 10-item Self-Repression Scale and the Emotional Social Support Scale (10 items for support from the family and 10 items for support from friends/others). ${ }^{34}$

\section{Statistical analyses}

Data are presented as mean values and SD, or frequency and percent. Categorical data were evaluated using the Chi-squared test. The Kruskal-Wallis and WilcoxonMann-Whitney tests were used to compare proportions among groups. For parametric data with normal distribution, Student's $t$-tests for paired or unpaired observations were used as appropriate. Associations between factors were tested by standard linear regression. Statistical significance was set at a $P$-value of $<0.05$. All statistical analyses were conducted with SPSS software (v. 11.5 for Windows; SPSS Japan Inc., Tokyo, Japan).

\section{Results}

\section{Distribution of endothelial function}

Of 411 subjects originally identified, a total of 74 subjects were considered eligible based on their health check-ups and completed the evaluations. No disease was observed in any of the subjects. The average reactive hyperemia (RH)-PAT index value of these subjects was $1.95 \pm 0.47$. Forty-nine subjects had an RH-PAT index value of $\geq 1.67$, and 25 subjects had an RH-PAT index value of $<1.67$. Accordingly, one-third of the subjects had RH-PAT values indicating endothelial dysfunction. No difference in RH-PAT index values was observed by sex (Figure 2A) or by age (Figure 2B).

The subjects with normal endothelial function were compared with those with endothelial dysfunction in terms of the following parameters: potential onset of risk factors, age, body mass index, blood markers, psychosocial assessment, lifestyle and vascular dynamics. None of these cardiovascular risk factors differed significantly between the two groups of 

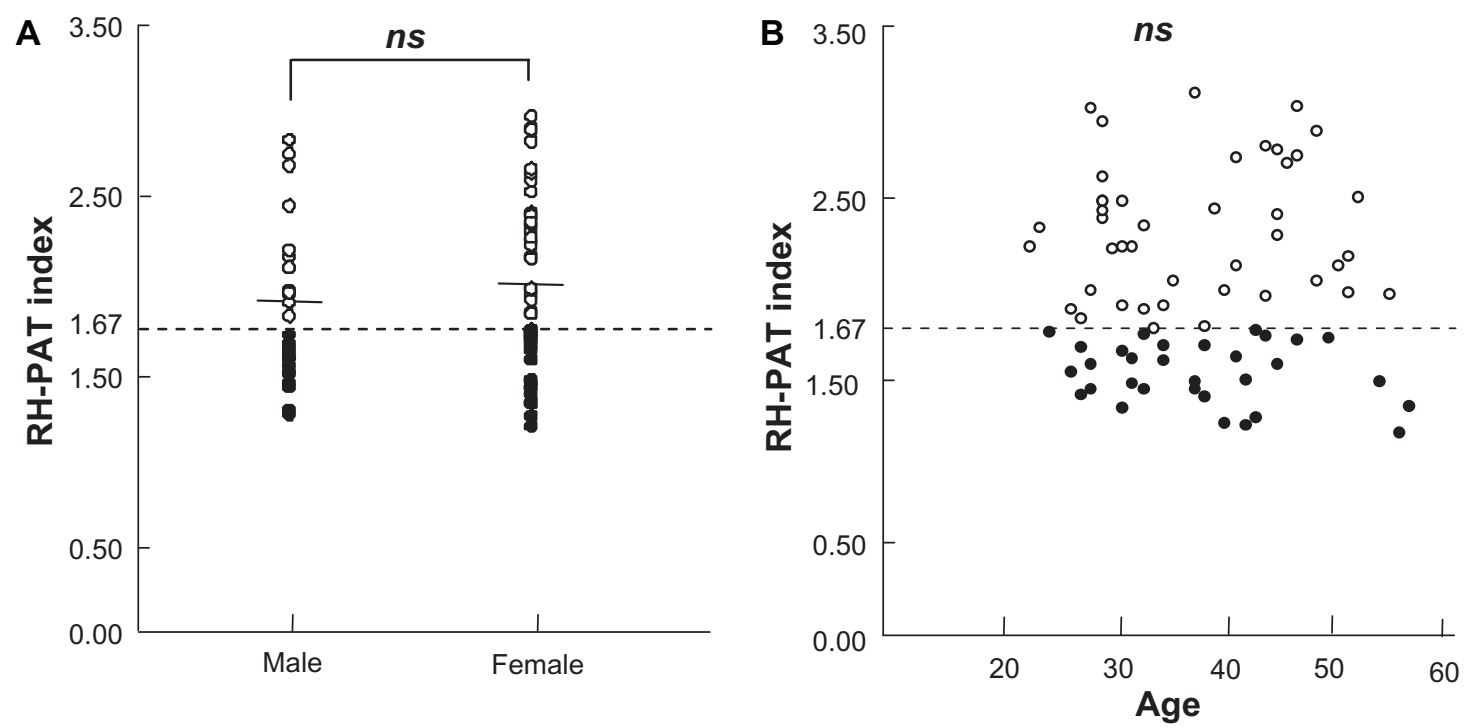

Figure 2 The distribution of endothelial function in 74 subjects considered to have normal health status in health check-ups. The open dots indicate RH-PAT index values of $\geq 1.67$ and black dots indicate RH-PAT index values of $<$ I.67. An index value of $<1.67$ was considered to indicate endothelial dysfunction (low response). Overall, $33.8 \%$ (25/74) of the healthy subjects had endothelial dysfunction. There were no significant differences nor correlation in RH-PAT index values between sexes A or age groups B. Abbreviations: ns, not significant; RH-PAT, reactive hyperemia peripheral arterial tonometry.

subjects (Table 2). Next, we measured endothelial function twice-daily in 19 of 25 subjects with an RH-PAT of $<1.67$. When comparing endothelial function after the second and third examinations, $15.8 \%$ of the subjects had RH-PAT index values above the standard value of 1.67 after both examinations, $26.3 \%$ of the subjects had values of $<1.67$ at both examinations, and $57.8 \%$ had values of $<1.67$ at least once. Seven of 74 subjects had familial histories of cerebro-cardiovascular disease including hypertension. There were no significant differences between these three groups in terms of risk factors for cardiovascular events.

\section{Association between relative fatigue and endothelial function}

There was non-significant change in the RH-PAT index when comparing the values before and after work in 19 subjects (Figure $3 \mathrm{~A} ; t=-1.13, P=0.275$ ). Similarly, there was no relationship between endothelial function and quantified fatigue (AFC value). However, the endothelial function values fluctuated significantly, along with subjective fatigue, at different times of the day (Figure 3B). The mean RH-PAT index value was $1.86 \pm 0.47$ in subjects reporting low subjective fatigue and $1.54 \pm 0.23$ in subjects reporting greater subjective fatigue ( $t=2.54, P=0.008)$, corresponding to a decrease of $-34.6 \%$ (Figure $3 \mathrm{~B}$ ). In other words, subjects with greater subjective fatigue showed a significantly lower endothelial function response. The reasons for subjective fatigue included domestic duties ( $(n=5)$, being out of shape $(n=5)$, a reason other than work itself $(n=7)$, or no reason $(n=2)$.
On the other and, there was a nonsignificant change in ba-PWV between the morning and the afternoon $(t=1.04$, $P=0.178)$. Furthermore, there was no association between endothelial function and duration of sleeping (data not shown). Moreover, endothelial function was not associated with AFC or psychosocial factors. However, associations were found among factors that potentially affect fatigue. There were negative correlations between AFC and Emotional Social-Support from Friends ( $\rho=-053, P=0.041$ ), between the Self-Esteem Scale and State-Trait Anxiety Inventory ( $\rho=-0.58, P=0.002$ ), between the Self-Esteem Scale and the Self-Repression Scale $(\rho=-0.73, P=0.002)$, and between the Self-Esteem Scale and the Self-Rated Depression scale $(\rho=-0.60, P=0.018)$ (Table 3).

\section{Discussion}

\section{Nontraditional risk factors of endothelial dysfunction}

Endothelial dysfunction is considered to be involved in the pathway between the development of atherosclerosis and the development of ischemic disease. ${ }^{18}$ Endothelial dysfunction progresses with increases in risk factors for ischemic heart disease, such as advanced age, smoking, physical inactivity, diabetes, dyslipidemia and hypertension. ${ }^{16,35,36}$ These risk factors exert a marked influence on endothelial cells over time and contribute to systemic manifestation such as CCVD and Sudden Cardiac Death. ${ }^{37}$

Conversely, several studies have reported that psychosocial factors affect CCVD, ${ }^{5-7}$ other than the above-mentioned 

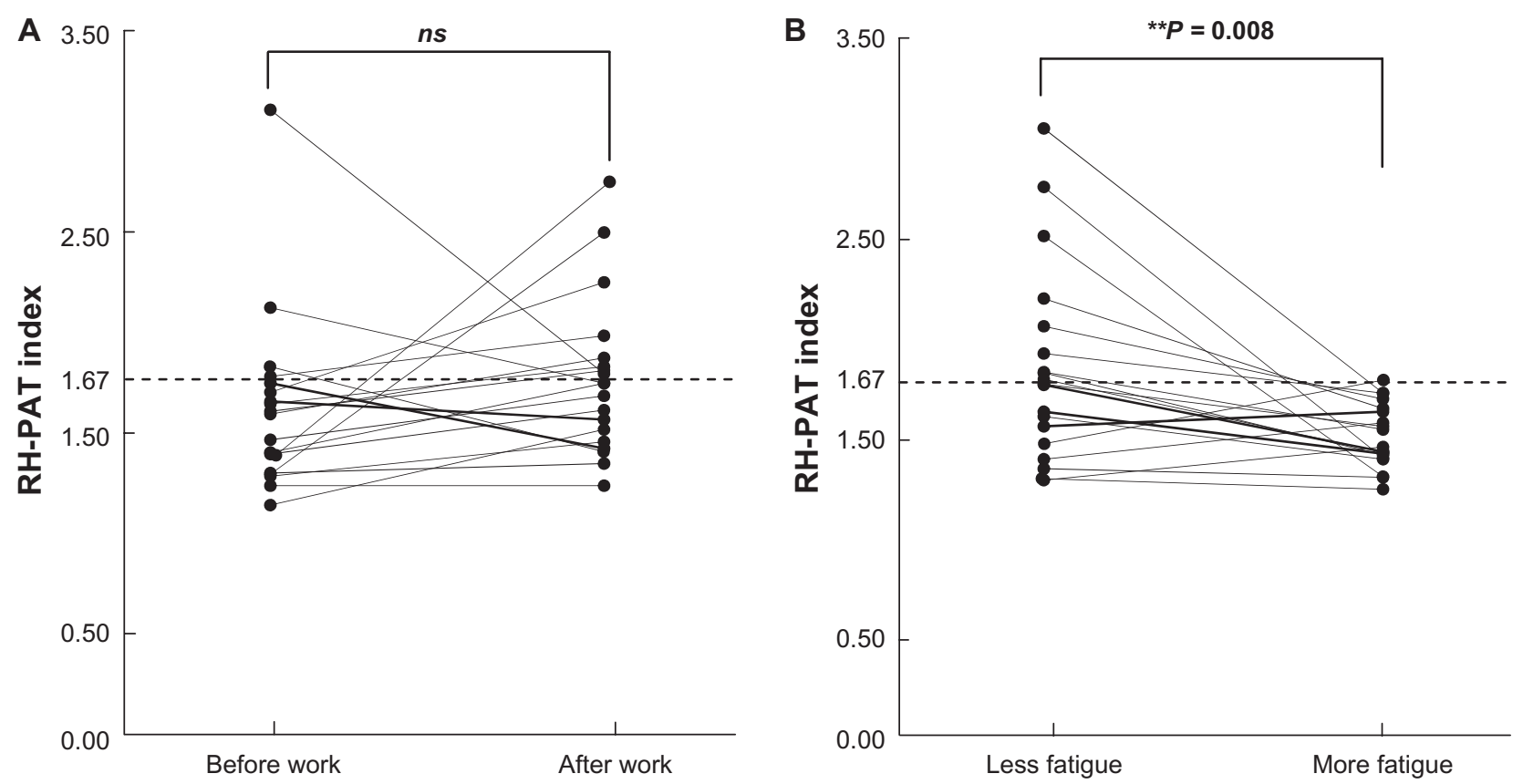

Figure 3 A) Effect of work on RH-PAT and endothelial function. For 19 subjects with a low response at the initial measurement (RH-PAT index values $<$ I.67), endothelial function was examined twice a day, before and after work. However, there was no difference in endothelial function before versus after work. **P $<0.0 \mathrm{I}$ vs before work. B) Effect of subjective fatigue on endothelial function. RH-PAT values in individuals with an initial low response were classified into subjects who reported more fatigue and subjects who reported less fatigue. Subjective fatigue had a significant effect on endothelial function. $* * P<0.01$ vs less fatigue.

Abbreviations: RH-PAT, reactive hyperemia peripheral arterial tonometry; ns, not significant.

traditional ischemic risk factors. ${ }^{8}$ Some FMD studies have revealed that there is no difference in the prevalence of these risk factors in subjects with normal endothelial function values versus subjects with endothelial dysfunction. ${ }^{8}$ In this study, one-third of the healthy subjects had PAT values of $<1.67$, indicating low-response endothelial dysfunction, which means that endothelial function is sometimes abnormal in healthy people. Furthermore, we showed that endothelial function fluctuates diurnally, with an interaction between the individual's cognitive fatigue and the individual's environment (Figure 4). Here, we found no diurnal variations in the pulse wave velocity. This was because we excluded patients with hypertension, and all of the subjects included in our study had stable blood pressure. Thus, we consider that endothelial function is more likely to reflect diurnal variations than pulse wave velocity. Although the ba-PWV reflects arterial stiffness and $\mathrm{ABI}$ reflects the occlusion of lower extremity arteries, both were within their normal ranges in this study.

Table 3 Correlations among endothelial function and fatigue related examination subscales

\begin{tabular}{|c|c|c|c|c|c|c|c|c|c|}
\hline & 2 & 3 & 4 & 5 & 6 & 7 & 8 & 9 & 10 \\
\hline I. 2nd endothelial function & 0.114 & 0.321 & -0.109 & 0.431 & 0.043 & -0.400 & 0.269 & -0.430 & -0.428 \\
\hline 2. 3rd endothelial function & & 0.155 & 0.252 & $0.04 I$ & -0.346 & 0.146 & -0.116 & 0.281 & 0.015 \\
\hline 3. AFC (accumulated fatigue checklist) & & & -0.091 & 0.345 & 0.390 & -0.363 & 0.219 & -0.179 & $-0.533^{*}$ \\
\hline $\begin{array}{l}\text { 4. Sleeping hrs (previous day of } 2 \text { nd } \\
\text { examination) }\end{array}$ & & & & 0.187 & -0.022 & 0.062 & 0.195 & 0.024 & 0.234 \\
\hline 5. Self-rating depression scale & & & & & 0.480 & $-0.599 *$ & $0.565^{*}$ & -0.404 & -0.270 \\
\hline 6. State-trait anxiety inventory & & & & & & $0.579 *$ & $0.77 I^{* *}$ & -0.155 & -0.046 \\
\hline 7. Self esteem scale & & & & & & & $-0.734 * *$ & 0.490 & 0.254 \\
\hline 8. Self repression scale & & & & & & & & 0.181 & -0.117 \\
\hline 9. Emotional social-support from family & & & & & & & & & $0.519 *$ \\
\hline 10. Emotional social support from friends & & & & & & & & & \\
\hline
\end{tabular}

Notes: Coefficients $(\rho)$ are shown. $\mathrm{n}=1$ 19. *Significant at $P<0.05$, **Significant at $P<0.01$. 


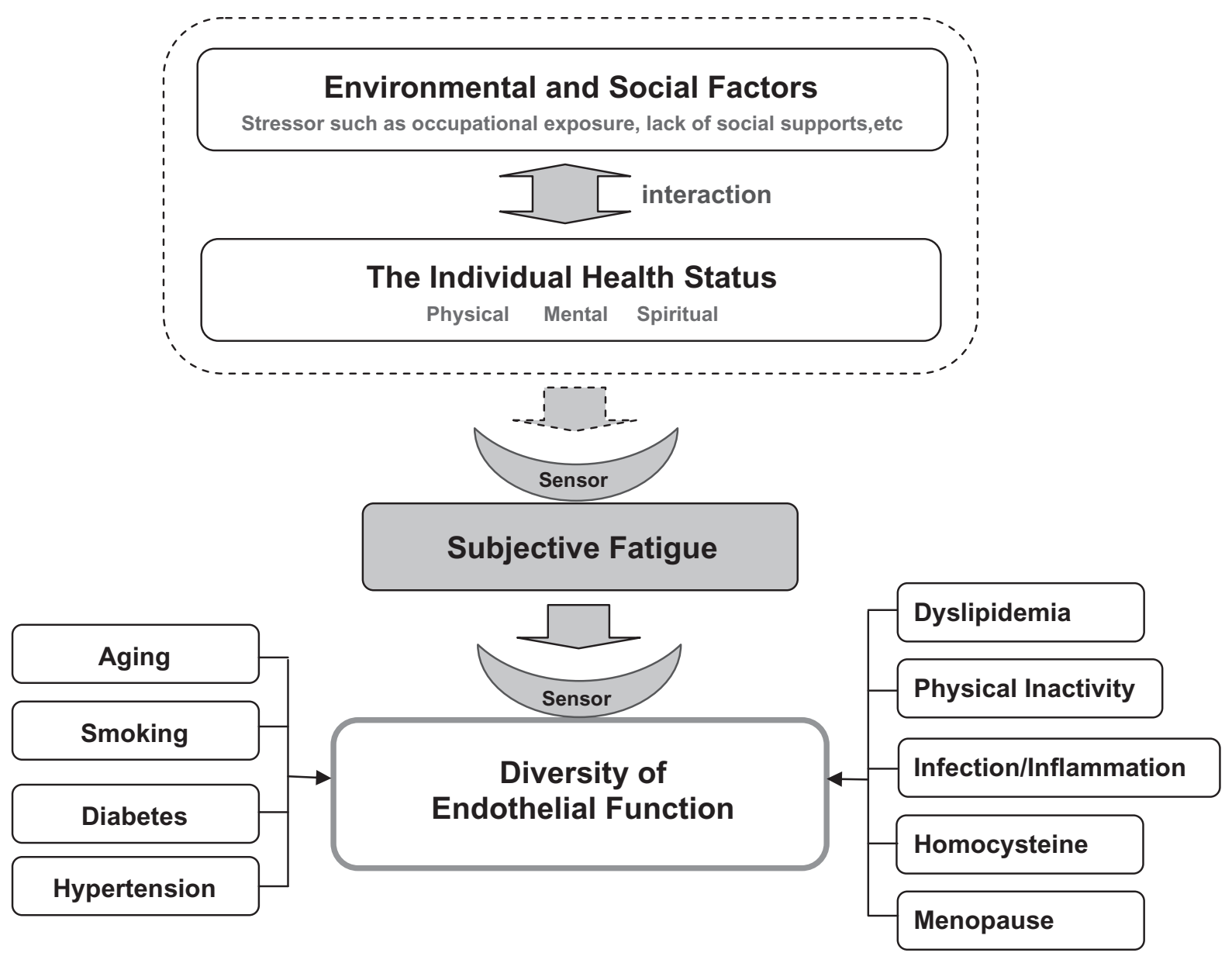

Figure 4 Endothelial function as a sensor of subjective fatigue. The health status of the individual is influenced by interactions between environmental and social factors such as occupational exposure and lack of social support. Subjective fatigue represents an integrated expression of an individual's physical, mental and spiritual condition. Endothelial function is influenced by subjective fatigue, which is a potential risk factor for endothelial dysfunction, independently of traditional cerebro-cardiovascular disease risk factors. Abbreviation: PAT, peripheral arterial tonometry.

This was considered to be due to the exclusion of individuals with traditional risk factors in this study.

\section{Fatigue and endothelial dysfunction}

Based on the epidemiological evidence that vital exhaustion and/or mental stress are associated with $\mathrm{CCVD},{ }^{1-4}$ the physiological processes of fatigue must affect vascular function. However, the mechanisms by which the vascular function of healthy subjects is influenced by changes in subjective fatigue or accumulated fatigue processes are still unclear. The results of this study are important in verifying one of these processes, namely that the strength of fatigue within one person is significantly associated with vascular function and could be considered as a risk factor for vascular dysfunction.

This study has shown that subjective fatigue is significantly associated with endothelial function (Figure 3). Endothelial function measured by PAT did not vary according to daily work, but rather to the level of subjective fatigue.
As vascular function is not only affected by cardiac function but also by the autonomic nervous system and the endocrine system, non-traditional risk factors, other than the recognized traditional risk factors represented by arteriosclerosis risk factors, are predicted to exist. In this study, we focused on one of the nontraditional risk factors, fatigue. It is worth noting that endothelial function varied significantly according to self-evaluated strength of fatigue. Furthermore, we also found that endothelial function is not only associated with risk factors for ischemic disease, but also fluctuated diurnally in healthy subjects. This might represent a total body reaction to the psychosocial interaction between the individual and the environment (Figure 4). Nevertheless, associations between fatigue and psychosocial factors such as depression, self esteem and anxiety have been reported ${ }^{24}$ and similar results were observed in this study (Table 3 ). Our observations suggest that endothelial dysfunction may be a risk factor for CCVD, even in healthy people. 


\section{Evaluation of fatigue}

Fatigue is commonly defined as a pervasive sense of tiredness or a lack of energy that is not exclusively attributable to exertion. ${ }^{38}$ However, a unified definition remains elusive, partly because fatigue is a complex phenomenon that involves a number of psychosocial, behavioral and/or physiological processes ${ }^{39}$ The levels of fatigue differ among individuals and absolute quantification is still difficult. In this study, we showed that fatigue affects endothelial function based on the relative fatigue assessment in individual participants. The temporal state of fatigue is conceptually distinct from the pathological state. ${ }^{2,25}$ The relative change in the level of fatigue after the second and third measurements was correlated with endothelial function. However, absolute quantification of fatigue with a single cognitive assessment using fatigue-related scales is difficult and there seemed to be no correlation between the individual fatigue-related scales and endothelial function. Moreover, although the etiology of burnout or vital exhaustion has been investigated and cohort studies have been conducted, ${ }^{2-4}$ it is still not possible to distinguish between pathological fatigue and physiological fatigue. It is important to establish an objective method of assessing fatigue. Although various approaches have been attempted, including animal models of fatigue, ${ }^{40,41}$ an objective method to measure physiological fatigue in healthy people has not yet been developed. Menopausal status did not affect the conclusion of our study because we evaluated the relationship between endothelial function and fatigue in individual participants. Although we only conducted further assessments in subjects who had shown abnormal endothelial function at the first examination in this study, we intend to extend our study to include all subjects to generalize the correlation between fatigue and endothelial function.

In this paper, we propose a new concept that vascular endothelial cells are a potential sensor of fatigue (Figure 4). The differences between the individuals' reaction to the environment is reflected in their health status. ${ }^{42}$ Fatigue represents a systemic reaction of the person and endothelial function seems to react sensitively as an objective sensor of fatigue. In terms of the clinical utility of measuring fatigue, acknowledging the individual's magnitude of fatigue at baseline is important to recognize how changes in level of fatigue leads to endothelial function and thus self-management.

\section{Conclusion}

Our study indicates that changes in the strength of fatigue could influence endothelial function, even in the absence of traditional risk factors for ischemic disease. We propose a new concept that vascular endothelial cells are a potential sensor of fatigue. Endothelial function, as an objective tool to measure fatigue in healthy subjects, could be used to aid selfmanagement or health education for healthy individuals.

\section{Acknowledgments/disclosures}

The authors report no conflicts of interest in this work. We wish to thank Takashi Mawatari, Director, and Kayoko Higashi, Nursing Manager, of the Kagoshima Seikyo General Hospital and all staff involved in this study.

\section{References}

1. Melamed S, Shirom A, Toker S, Berliner S, Shapira I. Burnout and risk of cardiovascular disease: evidence, possible causal paths, and promising research directions. Psychol Bull. 2006;132(3):327-353.

2. Soufer R, Jain H, Yoon AJ. Heart-brain interactions in mental stress-induced myocardial ischemia. Curr Cardiol Rep. 2009;11(2): 133-140.

3. Kop WJ, Appels AP, Mendes de Leon CF, de Swart HB, Bar FW Vital exhaustion predicts new cardiac events after successful coronary angioplasty. Psychosom Med. 1994;56(4):281-287.

4. Naess H, Nyland HI, Thomassen L, Aarseth J, Myhr KM. Fatigue at long-term follow-up in young adults with cerebral infarction. Cerebrovasc Dis. 2005;20(4):245-250.

5. Naess H, Waje-Andreassen U, Thomassen L, Nyland H, Myhr KM. Health-related quality of life among young adults with ischemic stroke on long-term follow-up. Stroke. 2006;37(5):1232-1236.

6. Alboni P, Favaron E, Paparella N, Sciammarella M, Pedaci M. Is there an association between depression and cardiovascular mortality or sudden death? J Cardiovasc Med (Hagerstown). 2008;9(4):356-362.

7. Ketterer MW, Knysz W, Keteyian SJ, et al. Cardiovascular symptoms in coronary-artery disease patients are strongly correlated with emotional distress. Psychosomatics. 2008;49(3):230-234.

8. Bonetti PO, Lerman LO, Lerman A. Endothelial dysfunction: a marker of atherosclerotic risk. Arterioscler Thromb Vasc Biol. 2003;23(2): $168-175$.

9. Huang AL, Silver AE, Shvenke E, et al. Predictive value of reactive hyperemia for cardiovascular events in patients with peripheral arterial disease undergoing vascular surgery. Arterioscler Thromb Vasc Biol. 2007;27(10):2113-2119.

10. Mitchell GF, Parise H, Vita JA, et al. Local shear stress and brachial artery flow-mediated dilation: the Framingham Heart Study. Hypertension. 2004;44(2):134-139.

11. Vane JR, Anggard EE, Botting RM. Regulatory functions of the vascular endothelium. N Engl J Med. 1990;323(1):27-36.

12. Esper RJ, Nordaby RA, Vilarino JO, Paragano A, Cacharron JL, Machado RA. Endothelial dysfunction: a comprehensive appraisal. Cardiovasc Diabetol. 2006;5:4.

13. Gibbons GH, Dzau VJ. The emerging concept of vascular remodeling N Engl J Med. 1994;330(20):1431-1438.

14. Benjamin EJ, Larson MG, Keyes MJ, et al. Clinical correlates and heritability of flow-mediated dilation in the community: the Framingham Heart Study. Circulation. 2004;109(5):613-619.

15. Orshal JM, Khalil RA. Gender, sex hormones, and vascular tone. Am J Physiol Regul Integr Comp Physiol. 2004;286(2):R233-R249.

16. Wang J, Widlansky ME. Lifestyle choices and endothelial function: risk and relevance. Curr Vasc Pharmacol. 2009;7(2):209-224.

17. Schachinger V, Britten MB, Zeiher AM. Prognostic impact of coronary vasodilator dysfunction on adverse long-term outcome of coronary heart disease. Circulation. 2000;101(16):1899-1906.

18. Barac A, Campia U, Panza JA. Methods for evaluating endothelial function in humans. Hypertension. 2007;49(4):748-760. 
19. Kuvin JT, Patel AR, Sliney KA, et al. Assessment of peripheral vascular endothelial function with finger arterial pulse wave amplitude. Am Heart J. 2003;146(1):168-174.

20. Yeo TW, Lampah DA, Gitawati R, et al. Impaired nitric oxide bioavailability and L-arginine reversible endothelial dysfunction in adults with falciparum malaria. J Exp Med. 2007;204(11):2693-2704.

21. Bonetti PO, Barsness GW, Keelan PC, et al. Enhanced external counterpulsation improves endothelial function in patients with symptomatic coronary artery disease. J Am Coll Cardiol. 2003;41(10):1761-1768.

22. Tiesinga LJ, Dassen TW, Halfens RJ. Fatigue: a summary of the definitions, dimensions, and indicators. Nurs Diagn. 1996;7(2):51-62.

23. Leone SS, Huibers MJ, Knottnerus JA, Kant IJ. Similarities, overlap and differences between burnout and prolonged fatigue in the working population. QJM. 2007;100(10):617-627.

24. Gielissen MF, Knoop H, Servaes P, et al. Differences in the experience of fatigue in patients and healthy controls: patients' descriptions. Health Qual Life Outcomes. 2007;5:36.

25. Dittner AJ, Wessely SC, Brown RG. The assessment of fatigue: a practical guide for clinicians and researchers. $J$ Psychosom Res. 2004;56(2):157-170.

26. Breslow L, Enstrom JE. Persistence of health habits and their relationship to mortality. Prev Med. 1980;9(4):469-483.

27. Kuvin JT, Mammen A, Mooney P, Alsheikh-Ali AA, Karas RH. Assessment of peripheral vascular endothelial function in the ambulatory setting. Vasc Med. 2007;12(1):13-16.

28. Yinon D, Lowenstein L, Suraya S, et al. Pre-eclampsia is associated with sleep-disordered breathing and endothelial dysfunction. Eur Respir J. 2006;27(2):328-333.

29. Bland JM, Altman DG. Statistical methods for assessing agreement between two methods of clinical measurement. Lancet. 1986;1: 307-310.

30. Sasaki T, Iwasaki K, Mori I, Hisanaga N, Shibata E. Overtime, job stressors, sleep/rest, and fatigue of Japanese workers in a company. Ind Health. 2007;45(2):237-246.

31. Shumway M, Sentell T, Unick G, Bamberg W. Cognitive complexity of self-administered depression measures. J Affect Disord. 2004;83 (2-3):191-198.
32. Serretti A, Olgiati P, Colombo C. Components of self-esteem in affective patients and non-psychiatric controls. J Affect Disord. 2005;88(1): 93-98.

33. Hishinuma ES, Miyamoto RH, Nishimura ST, et al. Psychometric properties of the state-trait anxiety inventory for Asian/Pacific-islander adolescents. Assessment. 2000;7(1):17-36.

34. Fukui S, Koike M, Ooba A, Uchitomi Y. The effect of a psychosocial group intervention on loneliness and social support for Japanese women with primary breast cancer. Oncol Nurs Forum. 2003;30(5):823-830.

35. Mensah GA, Ryan US, Hooper WC, et al. Vascular endothelium summary statement II: Cardiovascular disease prevention and control. Vascul Pharmacol. 2007;46(5):318-320.

36. Naomi M. Hamburg, Michelle J. Keyes, Martin G. Larson, et al. Cross-sectional relations of digital vascular function to cardiovascular risk factors in the Framingham Heart Study. Circulation. 2008:117:2467-2474.

37. Smith TW, Cain ME. Sudden cardiac death: epidemiologic and financial worldwide perspective. J Interv Card Electrophysiol. 2006;17(3): 199-203.

38. Brown RF, Schutte NS. Direct and indirect relationships between emotional intelligence and subjective fatigue in university students. J Psychosom Res. 2006;60(6):585-593.

39. Shen J, Barbera J, Shapiro CM. Distinguishing sleepiness and fatigue: focus on definition and measurement. Sleep Med Rev. 2006;10(1): 63-76.

40. Sener G, Paskaloglu K, Satiroglu H, Alican I, Kacmaz A, Sakarcan A. L-carnitine ameliorates oxidative damage due to chronic renal failure in rats. J Cardiovasc Pharmacol. 2004;43(5):698-705.

41. Vij G, Gupta A, Chopra K. Modulation of antigen-induced chronic fatigue in mouse model of water immersion stress by naringin, a polyphenolic antioxidant. Fundam Clin Pharmacol. 2009;23(3):331-337.

42. Gee GC, Payne-Sturges DC. Environmental health disparities: a framework integrating psychosocial and environmental concepts. Environ Health Perspect. 2004;112(17):1645-1653.
Vascular Health and Risk Management

\section{Publish your work in this journal}

Vascular Health and Risk Management is an international, peerreviewed journal of therapeutics and risk management, focusing on concise rapid reporting of clinical studies on the processes involved in the maintenance of vascular health; the monitoring, prevention and treatment of vascular disease and its sequelae; and the involvement of

\section{Dovepress}

metabolic disorders, particularly diabetes. This journal is indexed on PubMed Central and MedLine. The manuscript management system is completely online and includes a very quick and fair peer-review system, which is all easy to use. Visit http://www.dovepress.com/ testimonials.php to read real quotes from published authors. 